\title{
Comunidades Virtuales de Aprendizaje y trabajo Cooperativo: Una experiencia innovadora en la escuela "Gabriela Mistral"
}

Virtual Learning Communities and Cooperative work: An innovative experience in the "Gabriela Mistral" school

\author{
María Verónica Peralta-Ortiz \\ maria.peralta.97@est.ucacue.edu.ec \\ Universidad Católica de Cuenca, Azogues \\ Ecuador \\ https://orcid.org/0000-0002-3185-2172 \\ Darwin Gabriel García-Herrera \\ dggarciah@ucacue.edu.ec \\ Universidad Católica de Cuenca, Azogues \\ Ecuador \\ https://orcid.org/0000-0001-6813-8100 \\ Sandra Elizabeth Mena-Clerque \\ sandramena@ucacue.edu.ec \\ Universidad Católica de Cuenca, Cuenca \\ Ecuador \\ https://orcid.org/0000-0002-9186-2161
}

Recepción: 10 de abril 2021

Revisado: 05 de mayo 2021

Aprobación: 30 de junio 2021

Publicación: 15 de julio 2021 


\title{
RESUMEN
}

El presente trabajo aborda la incidencia de la implementación y ejecución de una Comunidad Virtual de Aprendizaje (CVA) para analizar los efectos del trabajo cooperativo en la construcción de objetivos comunes entre dos instituciones que pueden aprender una de la otra, primando el reconocimiento y respeto por las diversas culturas, sin que la limitación geográfica sea un impedimento para interactuar con personas de otros países y que ello tenga un trasfondo pedagógico, para ello, los miembros deben aportar de manera individual y generar un aprendizaje colectivo mediante el uso de herramientas digitales que permita construir ambientes virtuales de aprendizaje. La investigación tuvo un enfoque mixto aplicado de manera transversal resaltando que la cooperación incidió positivamente en la implementación de una CVA para generar aprendizajes en los participantes, por ello se propone pasos para implementar una CVA dentro de una institución educativa.

Descriptores: Método de enseñanza; enseñanza multimedia; aprendizaje active. (Palabras tomadas del Tesauro UNESCO).

\begin{abstract}
This work addresses the incidence of the implementation and execution of a Virtual Learning Community (CVA) to analyze the effects of cooperative work in the construction of common goals between the institutions that can learn one from another, prioritizing recognition and respect for The different cultures, without the geographical limitation is an impediment to interact with people from other countries and that they have a pedagogical background, for them, members must contribute individually and generate collective learning through the use of digital tools that allow build virtual learning environments. The investigation has a mixed approach applied in a transversal way, emphasizing that the cooperation had a positive impact on the implementation of a CVA to generate learning in the participants, because it proposes steps to implement a CVA within an educational institution.
\end{abstract}

Descriptors: Teaching methods; multimedia instruction; activity learning. (Words taken from the UNESCO Thesaurus). 


\section{INTRODUCCIÓN}

En la actualidad el planeta se encuentra atravesando por una crisis sanitaria, una pandemia que impactó a nivel mundial en lo económico, social, político, tecnológico; un cambio que trasendió a un nuevo estilo de vida; adaptandose a una "nueva realidad" y a convivir con una emergencia en el área de la salud, pero al unísono también afloró una revolución de las TIC masificando su uso y abriendose paso el uso de las herramientas digitales.

En América Latina este impacto tomó por sorpresa a los gobiernos inestables por la falta de recursos para asumir un gasto público de magnitudes abismales y sumado a ello la corrupción que acompañó las acciones de unos cuantos al especular costos de insumos, consecuencias que el pueblo latinoamericano tuvo que asumir y, para sumar los males, el dolor de perder a sus seres queridos ante la vista de gobernantes que no accionaron oportunamente.

Para Ecuador esta crisis agravó la credibilidad de una presidencia por malos manejos de recursos, especulaciones, falta de liderazgo, entre otras circunstancias; pero que los ministerios de gobierno tuvieron que asumir acciones que intenten dar respuesta a las necesidades que se incrementaban conjuntamente el número de víctimas de COVID-19. El Ministerio de Educación ecuatoriano tuvo que aventurarse a la virtualidad; un cambio de estrategia, que al inicio, fue un traspié por la resistencia al cambio y al poco uso de herramientas digitales; si bien, antes de la llegada de la pandemia, la tecnología jugaba un papel importante para el desarrollo y desenvolvimiento de la sociedad, pero se evidenciaba que se rehusaba, en ciertos grupos sociales, a utilizarla por diversos motivos como la edad, falta de recursos, falta de interés , resistencia, miedo al cambio, entre otros; motivos que ampliaban la brecha digital en nuestro país. Esta desigualdad social se puede evidenciar en el acceso al equipamento y en el uso de lo que ya se tiene al alcance, limitándose a la utilidad de las herramientas digitales en su forma más básica sin intentar explorar las diversas y extensas posibilidades que nos brinda. 
La educación tuvo que alinearse a este "boom" digital, con una nueva mirada a formas de enseñar y aprender; por ende al auge de la teconolgía y la virtualidad se inmiscuyó en la vida cotidiana de la mayoría de estratos sociales; se masificó el uso de herramientas digitales para estar acorde a las necesidades que se presenta como el teletrabajo, clases mediante el uso de plataformas sincrónicas y asincrónicas, reuniones virtuales, entre otras.

Es evidente que Ecuador tuvo que adentrarse en el mundo digital obligando, de cierto modo, a su uso como parte de la cotidianidad de las personas y el sistema educativo vio la necesidad de adaptarse a esta realidad, pese a que no contaba con recursos y equipamento necesarios adherido al poco conocimiento de las herramientas digitales tanto de docentes como de estudiantes de estratos sociale medio-bajo ya que se observó poco conocimiento del uso del correo electrónico, el internet era limitado o sólo accedía ciertos sectores, no se manejaba plataformas educativas, el personal no estaba altamente capacitado para asumir esta competencia; factores que jugaron en contra al querer implementar una educación en la era digital tardía para Ecuador.

En contraste con lo expuesto, algunos docentes consideraban que dichas herramientas digitales y aparatos tecnológicos eran distractores en el proceso de enseñanzaaprendizaje, llegando a ser habitual que se prohíba su uso en las instituciones educativas (Duarte-Duarte \& Jurado, 2008). No obstante, esta necesidad digital acarreó a que los estudiantes se esfuercen por adquirir la herramienta tecnológica que ayude a dar continuidad a sus estudios en la virtualidad, alineados a los disposiciones ministeriales vigentes en tiempos de pandemia principalmente con la modalidad "Aprendamos Juntos en casa".

Si bien, el Ministerio de Educación ecuatoriano ha ambicionado aproximarse al uso de las TIC desde la década anterior, sus intentos no han sido cimentados debido a las dificultades que se han descrito con antelación. En publicaciones expuestas por parte del MINEDUC expresa que en el ámbito educativo las TIC, aparentemente, han tenido un progreso considerable en el uso del Interner para acceder a la información y con ello se 
advierten nuevos escenarios para el proceso de enseñanza - aprendizaje que desafían a un intercambio de conocimiento inmediato por parte de docentes y estudiantes, lo cual permite que el aprendizaje se construya de manera colaborativa, crítica y reflexiva; propiciando un ambiente dinámico, flexible, pluripersonal y pluridimensional (MinEduc, 2012).

Para acceder a las TIC y a entornos virtuales de aprendizaje se debe tener en cuenta la accesibilidad, las habilidades tecnológicas de la comunidad educativa, el pensamiento social y cultural, la participación activa y la apropiación personal para el uso adecuado y propositivo; y un punto medular es que los docentes deben tener las destrezas necesarias para posibilitar que los estudiantes accedan a este tipo de escenarios educativos y formativos.

Para contrastar lo dicho anteriormente (Prensky, 2010), asevera que los estudiantes en la actualidad, por su condición de nativos digitales, disponen una preferencia muy concreta a la hora de procesar la información y por tanto, prefieren el trabajo en red, la ludificación de los procesos de enseñanza-aprendizaje y la multitarea; en consecuencia, se desea desarrollar el uso adecuado de las herramientas para que beneficie al conocimiento y manejo con el objetivo de aprender de manera autónoma y colectiva a la vez: por ello se ve la necesidad de las competencias digitales en los docentes ya que los estudiantes a los que guían provienen de una nueva generación que, en ocasiones, supera al profesor en el uso de las TIC, teniendo mayor facilidad en el acceso a información, datos y conocimientos que se encuentra en el ciberespacio.

Sin duda alguna, actualmente, las herramientas digitales han posibilitado nuevas formas de acceso a la educación, desde un trabajo auntónomo posibilitando el desarrollo creativo, pensamiento crítico y personal; hasta un trabajo cooperativo que permita un aprendizaje grupal. En tal virtud, se debe aprovechar que la era digital se encuentra en su máximo apogeo y valernos de los recursos tecnológicos que manejan los estudiantes para proponer e implementar una Comunidad Virtual de Aprendizaje (CVA) que permita la interacción entre sus miembros, fomentar el trabajo cooperativo para garantizar su 
funcionalidad y que cumpla los objetivos planteados con un sentido de pertenencia dentro de la comunidad.

La ubicuidad posibilitará que esta interacción se realice entre dos instituciones de diferente país para fortalecer lazos culturales y fomentar el respeto a la diversidad, razón por la cual, nace una alianza educativa entre la escuela Gabriela Mistral de la ciudad de Cuenca y Tessa International School de la ciudad de New Jersey como una oportunidad para innovar las metodologías de enseñanza-aprendizaje, que permita constuir un conocimiento colectivo mediante el uso de un blog a fin de dar un uso propositivo a las herramientas digitales; posteriormente realizar un análisis de su implementació y funcionalidad; a fin de determinar la incidencia del trabajo cooperativo en la implementación de la Comunidad Virtual de Aprendizaje.

\section{Referencial teórico}

Asumiendo que la era virtual y la tecnología educacional se encuentra en su máximo resplandor en el Ecuador, que ha obligado a acercarnos a la virtualidad, a la adquisición del equipamiento electrónico, a la conexión de internet y sobre todo a redirigir las prácticas educativas. Valiéndose de todo lo descrito, se ha implementado ambientes virtuales de aprendizaje que han marcado el proceso educativo, como parte de la innovación y sirviéndose de la tecnología para que sea aprovechada por los participantes. En este sentido, (Torres et al. 2012), aseveran que los docentes deben darles un buen uso a las nuevas tecnologías de la información y ello será, sin duda, una ventaja tanto para el profesorado como para los estudiantes, no obstante, se requiere un período de adaptación y práctica en el uso de las herramientas, pero esto puede ser superado con la planificación clara del uso de la plataforma y valorando el esfuerzo continuo del estudiante en el desenvolvimiento participativo. En tal virtud, se va aclarando que la práctica educativa en un entorno virtual beneficia el aprendizaje y haciendo que éste sea más perdurable y pueda ser aplicado a la vida diaria para ser sujetos competentes que la sociedad demanda. 
Ante lo mencionado, el Sistema Educativo ecuatoriano está creando reformas y acuerdos ministeriales para asentarse en el panorama actual en miras de lo que se espera de la educación en tiempos de virtualidad y de las expectativas de un perfil apto de los docentes para que lo afronten, incluso capacitándolos para asumir este reto de una virtualidad tardía; pero, existe un desfase entre la mejora educativa escolar que aporta la tecnología elevando las expectativas de cambio, y por otro lado, los avances limitados que hasta el momento se han conseguido (Cueva-Gaibor, 2020).

Por otro lado, (Moreira-Segura \& Delgadillo-Espinoza, 2015), comentan las herramientas digitales como las redes sociales, wikis y los blogs están creando espacios virtuales de aprendizaje no formal y las instituciones educativas formales, en su mayoría, no aprovechan de este recurso; teniendo claro que los medios digitales son flexibles y facilitan la combinación y aplicación de distintas metodologías. Lo que se puede suponer que se mantienen una brecha digital ya sea por la apatía o desinterés en el uso de las herramientas por parte de algunos actores de la comunidad educativa; también por la poca accesibilidad a la tecnología y a sus recursos; o por la falta de medios para hacerlo. La virtualidad por la que está atravesando el Sistema Educativo ecuatoriano ha generado que el paradigma de educación cambie dando una mirada directa a un enfoque conectivista y su importancia en la actualidad; así mismo, la virtualidad, asumiéndola adecuadamente, puede llegar a ser una práctica muy productiva y gratificante, tanto para el docente como para el estudiante; con una visión altamente receptiva y proactiva y asumiendo que el docente es parte de esta comunidad y no su principal actor MoreiraSegura \& Delgadillo-Espinoza, 2015),

Para respaldar lo dicho anteriormente, (Camacho, 2018), en la parte concluyente de su artículo enfatiza que una Comunidad Virtual de Aprendizaje, como herramienta para afianzar el proceso de enseñanza, generada e incorporada al sistema educativo hará imprescindible una visión analítica para su aplicación en las instituciones educativas y conllevará a realizar cambios en la educación formal, con una nueva mirada en la 
actualización de procedimientos y programas en las distintas instituciones, ya sea educativas como también en las formadoras de docentes.

Tomando en cuenta la necesidad de una capacitación digital para ser competentes en la nueva atmósfera educativa; siendo éste el momento oportuno para que pueda habilitarse y prepararse con las herramientas necesarias inherentes al aula, con el alumnado que forma parte de esta sociedad del conocimiento inmersos en el mundo digital y de las TIC y que pertenecen a distintas comunidades virtuales pudiendo aprovechar para que se genere una efectiva Comunidad Virtual de Aprendizaje.

Adentrándonos a una práctica directa podemos citar estudio realizado por (Morales, 2016), en donde se implementa una CVA en una escuela de Bogotá con estudiantes de octavo y noveno; a fin de analizar las interacciones sociales para el desarrollo de colectivos inteligentes; en el estudio realizado desde su implementación hasta la fase final se extrae las conclusiones en donde indica que al inicio representó dificultades en la comprensión de la CVA para posteriormente.

Luego de la experiencia, logran reconfigurar la expectativa al reconocerse los participantes como agentes activos y que pueden aprovechar de la tecnología para potenciar su proceso educativo; evidenciando que los estudiantes se posicionaron frente al aprendizaje y esta acción trascendió al uso de la informática y a la interacción social necesaria para propiciar el aprendizaje, lo que conlleva a que los roles y las jerarquías que hay en la escuela sean replanteadas para centrarse fundamentalmente en la actividad de aprendizaje.

Valiéndonos de los planteamientos de los distintos autores y de acuerdo con lo emanado en sus citaciones es menester aseverar que la implementación de una CVA traerá nuevos retos a los estudiantes que será beneficioso para su aprendizaje, también se plantea que no será una tarea fácil porque posiblemente se pueda dar una resistencia al cambio, pero el objetivo está en que tanto estudiantes y docentes aprovechen las herramientas digitales para potenciar el aprendizaje activo mediante un trabajo cooperativo. 
Como se ha enfatizado varias veces, la educación también no escapa de la virtualidad y se ha enraizado a tal punto que los docentes han tenido que reestructurar sus procesos de enseñanza-aprendizaje valiéndose el conexionismo ante la situación por la que se atraviesa. También se ha tomado en cuenta que el ser humano es, por naturaleza, un ser social que necesita de los otros para vivir en comunidad y apropiarse de ella; por ello se desea resaltar la importancia de la implementación de una CVA entre instituciones, en donde el aprendizaje se genere de la cooperación y participación dentro de la comunidad, con una meta claramente definida.

Enfocándonos en un panorama más amplio, en donde las expectativas de un mundo globalizado deben ajustarse a lo imprescindible de la época actual por ello es indispensable la adquisición de las habilidades sociales para que las personas sean capaces de cooperar con su entorno. Pero también se presenta un lado, menos esperanzador, que muestra una realidad paralela, y es que, la cultura de colaboración no se desarrolla como se espera, y es en donde la competitividad prima los procesos sociales, evidenciando un estatus jerárquico y la exclusión al menos capacitado o simplemente desvalorizando su accionar. Estas realidades palpables, y a la vez contradictorias, nos muestran la necesidad de que los individuos aprendan a cooperar y dejen de competir; recayendo en los centros educativos, un papel fundamental, el de formar ciudadanos que la sociedad requiere, para respaldar lo descrito se cita a (Moriña, 2011), que afirma que no solo es necesario cooperar para aprender, sino que inicialmente se debe aprender a ser cooperativo.

\section{Cooperación en la educación}

El ser humano se interrelaciona como parte de la dinámica social, por ello debe adaptarse a las normas que la sociedad demanda para que haya armonía en la convivencia diaria. El arrimar hombro a hombro para conseguir un objetivo es parte de dicha dinámica y se ha desarrollado a lo largo de la historia, un ejemplo claro es la minga que nuestros antepasados acuñaban. 
El trabajo cooperativo esta inherente a la educación y la educación inmersa en la cooperación; un ejemplo concreto de cooperación educacional es cuando se debe elaborar los documentos institucionales (planes de mejora, código de convivencia, propuesta pedagógica, visión y misión institucional, etc.) en donde personal administrativo y cuerpo docente deben organizarse y apoyarse para llegar a los objetivos y facilite el intercambio de experiencias educativas.

Las ventajas del apoyo cooperativo entre docentes son diversas, es por ello que personal con trayectoria pueden guiar la práctica educativa a docentes que ingresan como novatos a las aulas de clases, y éstos, a su vez, pueden empapar con ideas frescas y renovadas; desde todas las direcciones, cuando se trabaja en equipo, los beneficios se desbordan a la hora de ejemplificar las posibilidades de compromiso cooperativo, cada persona desde su individualidad aporta para una mejora colectiva que beneficiará al conglomerado al que pertenece

En los salones de clases el apoyo cooperativo es fundamental entre estudiantes posibilitando una mayor interacción entre los sujetos y fortaleciendo el proceso educativo, dejando claro que el estudiante aprende de los conocimientos que brinda el docente y también con la interacción propositiva con sus compañeros de tal modo que los estudiantes son aprendices pero también mediadores de conocimiento; el desenvolvimiento individual se enriquece por la interacción con su entorno social en donde también se aprende de los otros pasando a ser generadores de un conocimiento constructivo.

Se considera al Aprendizaje Cooperativo (AC) como una metodología activa en los procesos de enseñanza-aprendizaje; privilegia la acción cooperativa en donde cada miembro debe aportar en la ejecución del trabajo para alcanzar una meta común, en otras palabras, el logro del integrante depende del logro del resto de miembros del equipo; por lo tanto, se vuelve un trabajo conjunto en donde se maximiza el aprendizaje propio y el de sus compañeros (Johnson \& Johnson, 2014). 
Se exhorta que el trabajo cooperativo no solo desea beneficiar al aprendizaje académico, sino a desarrollar habilidades sociales fomentado en el respeto, tolerancia, sentido de pertenencia de cumplimiento y ayuda a los demás, aceptación de las ideas ajenas, entre otras fortalezas; y ello, consecuentemente desarrollará habilidades individuales como la autoestima, autocontrol, autoimagen y sentirse útil para los demás.

El aprendizaje cooperativo se puede concebir como una teoría didáctica pero, a su vez, como una filosofía para guiar el proceso educativo y en su práctica involucra al docente debido a que facilita la conformación de grupos para propiciar la cooperación al trabajar y aprende juntos; y de cierta manera, promueve la implementación de comunidades de aprendizaje gobernadas por principios de cooperación y autonomía (Escorcia \& Gutiérrez, 2009).

\section{Virtualidad en la Educación}

La virtualidad de la educación acompañada de las posibilidades que ofrece el internet, ha dado un cambio del paradigma como lo ha sido a la largo de la historia con las herramientas que se presentaban en su momento como el periódico, la radio, el televisor, la computadora, etc. avances tecnológicos que se presentaron en determinada época y que revolucionaron en todas las direcciones, incluida la educación; que se ha beneficiado para innovar los procesos educativos. Debemos tener claro que en la educación se enfrenta a la "realidad" con la "virtualidad", que se ha querido apreciar como oponentes, pero no existe tal rivalidad; y ello se enraíza debido a que la educación radica en el proceso de socialización de los integrantes de una comunidad, con el objetivo de integrarlos en valores y normas imperantes en su proceso; en tal sentido, se entiende que toda educación es enseñanza y aprendizaje en la modalidad que se presente.

Se intenta, entonces, involucrar la virtualidad en los procesos educativos para aprovechar de los recursos que brinda, concordando con (Sangrá, 2001), quien no concibe que educación virtual y educación en línea sean sinónimos; el autor esboza la comprensión de educación virtual y la interrelación de sus elementos; enfatizando que educación y 
virtualidad se interrelacionan complementándose en la medida en la que la educación se vale de la creatividad de la virtualidad para optimizar y variar el proceso de enseñanzaaprendizaje y las acciones encaminadas a ella; en tanto que la virtualidad, como parte del sistema, se enriquece de los procesos metodológicos educativos y de comunicación, por ello, la finalidad esencial de ésta interrelación es que vaya más allá del simple acceso a la información.

Se desea que la virtualidad en la educación contribuya al proceso educativo, pero también es importante educarse en la virtualidad y posibilite al usuario utilizar las bondades que ofrece las TIC y el acceso a la web; (Soriano, 2015), hace diferencias entre el uso, el consumo y producción de los recursos e información en la educación para la virtualidad; lo que concerniría al "uso" se refiere a la instrumentación de los recursos (correos electrónicos, plataformas, aulas virtuales), en otras palabras, se limita al uso instrumental de recursos que pueden facilitar el proceso de enseñanza pero no significa que sea generador de aprendizaje; el "consumo" alude a la interactividad y a la toda la información que se puede acceder mediante el internet (blogs, redes sociales, chats, foros, revistas, etc.).

Por último, la "producción" se direcciona al diseño y creación de productos digitales por parte del usuario y que están disponibles en la virtualidad creados con posibilidad de ser consumidos por otros ciber navegantes. Por lo tanto, la virtualidad de la educación está determinada por lo flexible, abierta y disponible en cualquier momento y en cualquier lugar; no es rígido sin que intervenga el espacio temporal; es decir; lo simbólico y lo imaginario traspasa fronteras formando sociedades de conocimiento (Carpy, 2012). 


\section{Comunidades Virtuales de Aprendizaje (CVA)}

El término "comunidad", en su sentido más amplio, se lo puede definir como un conjunto de personas con interés y propósitos comunes; y en base a lo que expone (Locke, 2020), el reconocimiento y la importancia de comunidad es vital en el desarrollo de las comunidades virtuales, porque va más allá de un simple acto de conexión, su importancia radica en la interacción de las personas usando una amplia gama de dispositivos informáticos.

\section{Para comprender la funcionalidad de una CVA se realiza un breve análisis de lo que se entiende por Comunidades Virtuales (CV):}

La CV constituye una zona de acceso a la información teniendo el ciberespacio como escenario principal donde no hay territorio y cualquier persona, en cualquier momento pueda acceder; dando posibilidad a encuentros educativos, culturales y sociales; esta forma de aprehender en sociedad en red demanda el desarrollo de la inteligencia colectiva. (Cabero-Almenara, 2006), afirma que las CV son comunidades de individuos que participan compartiendo intereses comunes y su interacción es mediante las diversas herramientas de comunicación que nos brindan las redes telemáticas de manera sincrónica o asincrónica.

Partiendo de la expansión del internet en los años 80 se registra intentos de implementar CV en Estados Unidos en centros de investigación y académicos, iniciando una experiencia bidireccional mediante la red lo que posibilitaba una forma de gestionar y producción de la información. La implementación de una CV debe partir con un objetivo definido además de contar con el conjunto de personas con el firme propósito de perseguir dicho objetivo a través de intercambios, de una organización en su espacio virtual, una metodología de trabajo definida con archivos claros y con una jerarquía organizacional; sin tomar en cuenta el servicio que ofrece, los elementos indispensables para su funcionamiento son la colaboración, la participación activa, y el intercambio; a fin 
de generar un sentido de pertenencia de sus miembros necesario para que la comunidad evoluciones y se mantenga (Gairín, 2006).

Una CVA se implanta cuando el proceso educativo se sirve de una CV planteándose objetivos claros que estén al servicio del aprendizaje; el desafío es conseguir que las herramientas tecnológicas y digitales actuales se complementen con el proceso educativo posibilitando la interacción para crear entornos virtuales de aprendizaje, pero con un sentido de pertenencia entre sus miembros. Por tanto, se debe asumir que las TIC no son suficientes para que una CVA sea funcional, no obstante, aporta en gran medida a su implementación; lo que conlleva a pensar que el aprendizaje no se genera por el alcance a lo tecnológico sino por la apropiación de sus miembros desde un responsabilidad individual y colectiva a la vez para propiciar y desarrollar aprendizajes partiendo de una propuesta pedagógica que guiará el proceso.

Por consiguiente, (Carlén, 2002), define a las CVA como un conjunto de individuos que poseen intereses comunes y su aprendizaje es de manera colaborativa mediante el uso de las TIC. La definición anterior concuerda con lo que expresa (Lamí-Rodríguez-del-Rey et al. 2016), afirmando que es un conjunto de personas que interactúan de forma virtual teniendo intereses comunes y es parte del proceso educativo, cuya finalidad principal es el aprendizaje y el desarrollo profesional; entre sus miembros hay colaboración, intercambio de información y comunicación.

En un sentido más amplio, en la actualidad las CVA intenta crear un entorno para el aprendizaje que no se limite a recrear aulas formales ni creador de contenidos, sino que se fomente la interacción con espacios de comunicación que permita llevar a cabo las actividades, distribución de contenidos, elaboración de recursos educativos propios, acompañado de la tutoría y el soporte técnico; rompiendo limitaciones geográficas, horarios y el número de integrantes de un grupo (Camacho, 2018).

En base a lo expuesto, se puede afirmar que las CVA posibilita las interacciones con miembros de una misma institución o de diferentes entornos educativos, logrando un trabajo cooperativo y colaborativo de sus integrantes que participan de manera individual, 
pero al mismo tiempo integrados a un grupo, dicho de otro modo, la autonomía se nutre de la interdependencia y viceversa. Esto posibilita que, por una parte, se desarrolle o se cimente habilidades sociales y comunicativas, y por otra, que se use o se adquiera el manejo de recursos tecnológicos y digitales; pero, sobre todo, su implementación, estructura y funcionalidad debe satisfacer la finalidad propuesta y sus resultados deben garantizar el aprendizaje; considerando métodos apropiados de intervención, así como mecanismos de diagnóstico y evaluación permanente que permitan instaurar una mejora continua.

\section{METODOLOGÍA}

La presente invetigación tuvo un diseño metodológico no experimental con una tipología descriptiva, con enfoque mixto, desarrollada en la escuela "Gabriela Mistral" con estudiantes de quinto matutino teniendo 34 estudiantes matriculados en el período 20202021, estableciendose una muestra estratificada, en el proceso se recolecta 33 datos cuantitativos teniendo un nivel de confianza del $99 \%$ y un margen de error del $5 \%$.

Para la obtención de datos cuantitativos se hizo la intervención a los 33 estudiantes que se conectan de manera permanente al proceso educativo utilizando el cuestionario con 34 ítems para encuestarlos de forma guiada a través de la plataforma Zoom en un día y hora asignada, por el cual se envió el link del formulario realizado en Microsoft forms el mismo día y hora destinado para la encuesta, a fin de responder las dudas que se generaban con respecto a las preguntas y evitar que las respuestas sean duplicadas, cabe mecnionar que dicho instrumento fue validado por el método Delfhi

Como antesala se manifesta que los padres de familia fueron informados sobre la implementación de la CVA antes de ejecutarla, mediante una reunión a través de la plataforma ZOOM, en donde se comunicó varios puntos y en el orden del día constaba el proyecto de la CVA, porsteriormente fueron informados al grupo de representnates mediante WhatsApp como parte de las resoluciones establecidad en la reunión, adicionamente se ha pedido consentimiento para aplicar la encuesta mediante Microsoft 
Forms y, en algunos casos, vía telefónica a los representantes que no dieron respuesa al formulario de consentimiento informado.

Los datos cuantitativos recopilados fueron analizados mediante el sofrware IBM SPSS V.19, para su validación se aplicó el coeficiente de Alfa de Cronbach obtendiento un puntaje del 0,76 evidenciando que es una encuesta confiable. Se analizó las variables del cuestionario en base a la prueba de normalidad Shapiro Wilk obteniendo un nivel de significancia del 0,00 revelando que son paramétricas por ser menor al 0,05.

En cuanto a la obtención de datos cualitativos, se aplicó una entrevista semiestucturada con preguntas guía que incluía los aspectos abordados en la CVA a 3 docentes, una fue programada vía ZOOM a las 2 profesoras de la escuela de Estados Unidos del nivel prekinder que particiaron en la CVA, la otra entrevista se la realizó de forma escrita a la profesora del quinto de básica vespertino de la escuela Gabriela Mistral debido a que no se pudo coincidir en los horarios para hacerlo de maner directa. Los datos cualitativos recolectados se analizaron mediante una tabla de segmentación para describirlos de manera objetiva.

Tabla 1.

Instrumentos de recolección de datos aplicados en la investigación.

\begin{tabular}{|c|c|c|}
\hline Dimensión/enfoque & Cuantitativo & Cualitativo \\
\hline Población a aplicar & $\begin{array}{l}33 \text { estudiantes de quinto que se } \\
\text { conectan de manera permanente al } \\
\text { proceso eductaivo. }\end{array}$ & $\begin{array}{l}3 \text { Docentes que participaron en la CVA; } 2 \\
\text { docentes de Estados Unidos de prekinder } \\
\text { y } 1 \text { docente de quinto de básica jornada } \\
\text { vespertina de la escuela Gabriela Mistral. }\end{array}$ \\
\hline Técnica & $\begin{array}{l}\text { Encuesta guiada establecida con la } \\
\text { escala de Likert. }\end{array}$ & $\begin{array}{l}\text { Entrevista semiestructurada con preguntas } \\
\text { de diversas temáticas aplicadas en la CVA. }\end{array}$ \\
\hline Instrumento & $\begin{array}{l}\text { Cuestionario con preguntas cerradas } \\
\text { que constó de } 34 \text { ítem realizado en } \\
\text { Microssoft Forms. } \\
\text { Aplicado de manera colectiva } \\
\text { mediante zoom }\end{array}$ & $\begin{array}{l}\text { Protocolo de entrevista aplicada de manera } \\
\text { sincrónica a dos profesoras de Estados } \\
\text { Unidos mediante zoom. Una entrevista } \\
\text { escrita a la docente de Ecuador. }\end{array}$ \\
\hline
\end{tabular}

Elaboración: Los autores. 


\section{RESULTADOS}

En el proceso investigativo de la implementación de la CVA se observó de que manera incidió el trabajo cooperativo en la ejecución de la misma por ello se realizó el estudio desde el enfoque cualitativo y cuantitavo que nos permite desarrollar una estadística descriptiva a fin de dar a conocer sus resultados.

Partiendo del enfoque cualitativo, en la entrevista realizada a las docentes que participaron en la CVA de manera directa e indirecta se observó la incidencia que tuvo el trabajo cooperativo en la implementación CVA, siendo un factor importante a la hora de ejecutarlo.

En la siguiente tabla se muestra los aspectos relevantes que se tomaron de la entrevista:

\section{Tabla 2.}

Tabla de segmentación de la entrevista.

\begin{tabular}{|c|c|c|}
\hline Unidad de análisis & Categoría & Segmento \\
\hline $\begin{array}{l}\text { Implementación de una } \\
\text { CVA en los procesos } \\
\text { eductivos }\end{array}$ & Importancia/Utilidad & $\begin{array}{l}\text { Los entrevistados coiciden que la implementación de la CVA } \\
\text { fue útil en los procesos educativos. }\end{array}$ \\
\hline \multirow[t]{2}{*}{$\begin{array}{l}\text { Temas y actividades de la } \\
\text { comunidad educativa }\end{array}$} & $\begin{array}{l}\text { Fortalecimiento } \\
\text { conocimientos }\end{array}$ & $\begin{array}{l}\text { Los niños se interesaron por los temas. } \\
\text { Les ayudó a conocer otras palabras y la cultura. } \\
\text { Conocimientos a nivel cultural. }\end{array}$ \\
\hline & $\begin{array}{l}\text { Interés por conocer } \\
\text { manifestaciones } \\
\text { culturales }\end{array}$ & $\begin{array}{l}\text { Los niños se mativaron al tener el contacto directo con las } \\
\text { culturas que hay más allá. } \\
\text { Les sirvió como plataforma para que exploren más } \\
\text { alternativas y no sólo a ver las culturas de sus familias. } \\
\text { Les ha despertado el interés y la curiosidad al relacionarse } \\
\text { escuelas de dos distintos países. }\end{array}$ \\
\hline \multirow[t]{2}{*}{ Trabajo cooperativo } & Beneficio/utilidad & $\begin{array}{l}\text { Es necesario el trabajo cooperativo. } \\
\text { Fue útil cooperar. } \\
\text { Si fue útil, dejando atrás la rutina. }\end{array}$ \\
\hline & Actividades realizadas & $\begin{array}{l}\text { Los videos que se realizaron ayudaron al intercambio de } \\
\text { conocimientos. } \\
\text { Las maestras se apoyan de las ideas brindadas. } \\
\text { Mediante el trabajo cooperativo ha permitido el desarrollo de } \\
\text { distintas actividades y sus aportes han sido parte de sus } \\
\text { avences educativos. }\end{array}$ \\
\hline
\end{tabular}




Comunid Virtual de $\begin{aligned} & \text { Implementación } \\ & \text { permanente }\end{aligned}$
Aprendizaje
personal
Si vuelven a intentar con edades equilibradas, con objetivos más sólidos y que resultados esperar, tomar lo bueno de la presente implementación sería algo muy beneficioso. Sería una buena idea con estudianes que ya aporten con el vocabulario de mayor edad.

Sería de gran aporte a nivel institucional para tener una experiencia social y de aprendizaje mucho más completa al compartirla con todos los miembros.

Si recomendaría pero que la escuela se esfuerce por llegar a más niños, proveer de un espacio definido y con la ayuda de los padres de familia.

Si recomienda su implementación como proceso de enseñanza.

Recomienda al ser un espacio educativo que nos permite la interacción con otras personas y el uso de las herramientas tecnológicas que están ya inmersos en la práctica educativa.

Fuente: Entrevista.

En la entrevista los docentes manifestaron que fue muy útil esta innovación en los procesos educativos ya que los estudiantes se interesaron por los temas propuestos y les ayudó a conocer otra cultura de manera directa despertando su interés para explorar las alternativas propuestas mediante las herramientas que se fusionaron para que estén al alcance de los miembros de la comunidad y facilitar el aprendizaje; pero para que esta implementación haya tenido buenos resultados fue necesario el trabajo cooperativo entre docentes aportando con ideas y desarrollando actividades acorde al tema para integrarlos a una planificación de clases de las dos instituciones a fin de conseguir objetivos comunes de aprendizaje. Manifestaron también que la implementación permanente de una CVA sería una buena estrategia, tomando los aspectos positivos para mejorar su implementación con estudiantes que estén en edades equilibradas, que la escuela brinde espacios definidos para que pueda estar inmerso a la práctica educativa y que su implementación esté al alcance de la totalidad de sus estudiantes.

En cuanto al análisis cuantitativo, los resultados obtenidos reflejan en números concretos el efecto que tuvo la implementación de la CVA como proceso innovador en la escuela "Gabriela Mistral", ya que la encuesta fue dirigido al grupo de quinto de básica jornada matutina y los resultados se aprecian en las siguientes tablas: 
Tabla 3.

Incidencia de la CVA en el aprendizaje y el trabajo cooperativo.

\begin{tabular}{|c|c|c|c|c|c|c|c|}
\hline & & $\begin{array}{l}\text { CVA Refuerza } \\
\text { Aprendizaje }\end{array}$ & $\begin{array}{l}\text { Mejora de } \\
\text { aprendizaje } \\
\text { con TIC }\end{array}$ & $\begin{array}{l}\text { Interacción } \\
\text { mejoró uso } \\
\text { de TIC }\end{array}$ & $\begin{array}{c}\text { Interacción } \\
\text { con personas } \\
\text { otro país }\end{array}$ & $\begin{array}{l}\text { Conocer } \\
\text { culturas }\end{array}$ & $\begin{array}{c}\text { Fomentar } \\
\text { Trabajo } \\
\text { cooperativo }\end{array}$ \\
\hline \multirow[t]{2}{*}{$\mathrm{N}$} & Válidos & 33 & 33 & 33 & 33 & 33 & 33 \\
\hline & Perdidos & 0 & 0 & 0 & 0 & 0 & 0 \\
\hline \multicolumn{2}{|c|}{ Media } & 4,15 & 4,15 & 4,27 & 4,03 & 4,27 & 4,30 \\
\hline \multicolumn{2}{|c|}{ Mediana } & 5,00 & 5,00 & 5,00 & 4,00 & 5,00 & 5,00 \\
\hline \multicolumn{2}{|c|}{ Moda } & 5 & 5 & 5 & 5 & 5 & 5 \\
\hline \multicolumn{2}{|c|}{ Desv. típ. } & 1,202 & 1,253 & 1,306 & 1,311 & 1,153 & 1,015 \\
\hline \multicolumn{2}{|c|}{ Mínimo } & 1 & 1 & 1 & 1 & 1 & 2 \\
\hline \multicolumn{2}{|c|}{ Máximo } & 5 & 5 & 5 & 5 & 5 & 5 \\
\hline
\end{tabular}

Fuente: Encuesta.

En la anterior tabla los datos obtenidos demuestran que el aprendizaje mejora significativamente con la implementación de la CVA, y se enfoca directamente en el refuerzo del aprendizaje y el respaldo que brinda las TIC para que pueda darse dichos aprendizajes, el promedio indica un valor significativamente alto en estos aspectos descritos que relaciona al aprendizaje con la CVA. El trabajo cooperativo, que posibilitó la interacción con personas de otro país y conocer culturas, tiene una promedio bastante significativo, asumiendo la importancia de la cooperación en una CVA.

Se ha deseado interpretar la incidencia de una CVA a futuro y su aceptación para deteminar si la implementación sería una estrategia de enseñanza-aprendizaje valiéndose de los recursos digitales que ha tenido gran aceptación en los estudiantes como se evidenció en la tabla 3.

La siguiente figura muestra los indices de aceptación de los estudiantes de quinto matutino de la escuela Gabriela Mistral en cuanto a la aprobación de la CVA 
implementada durante los meses de marzo, abril y mayo del 2021; para establecer un criterio en cuanto a la implementación de manera permanente y en diferentes temáticas.
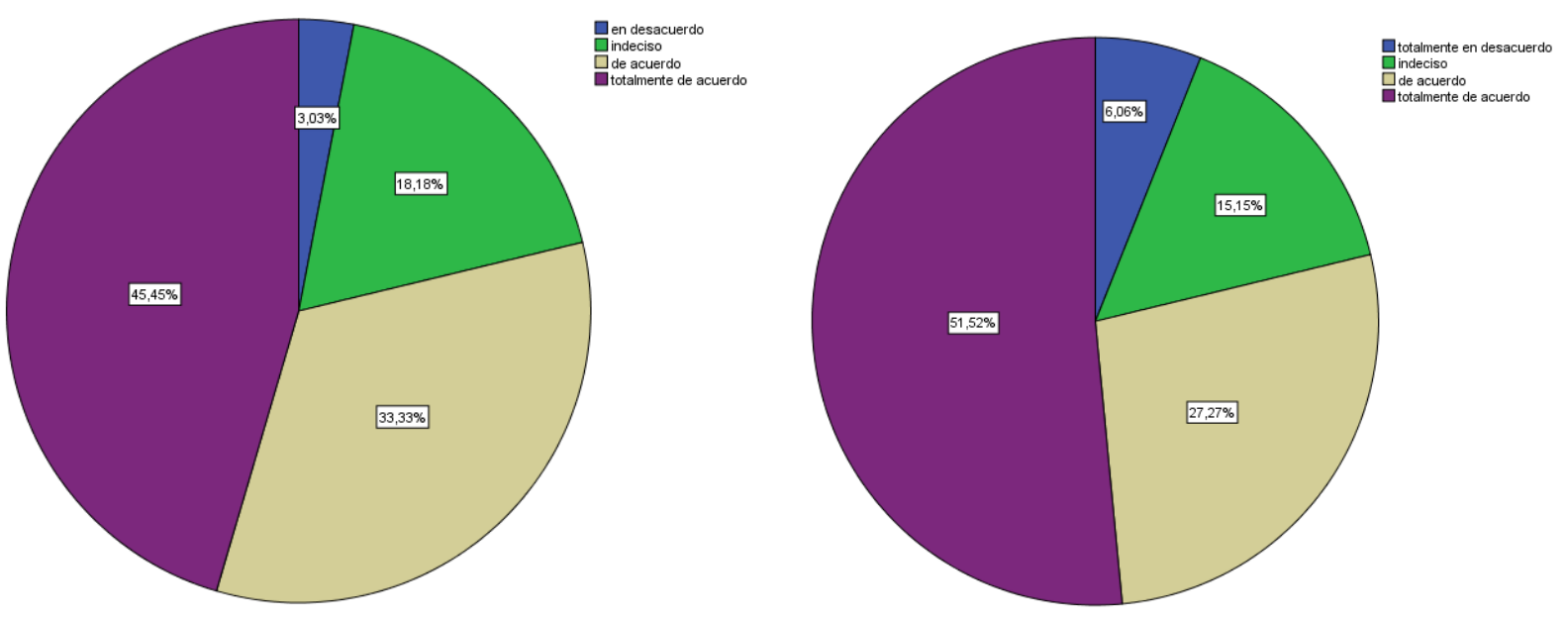

Figura 1. Gráfico de relación para que una CVA sea implementada demanera permanentes y en diversad temáticas.

Fuente: Encuesta.

Se aprecia que sumando los porcentajes de frecuencia "de acuerdo" y "totalmente de acuerdo" en cuanto a una implementación permanente nos da un total de $78,79 \%$ teniendo un nivel de aceptación relativamente alto. En cuanto a la aceptación de que una CVA sea implementada en diversas temáticas sumando las mismas frecuencias nos da un total de $78,78 \%$ obteniendo un porcentaje alto evidenciando la incidencia que tuvo la CVA implementada, es menéster mencionar que la aceptación de una CVA en diversas temáticas la frecuencia "totalmente de acuerdo" corresponde a más de la mitad del porcentaje con respecto a la sumatoria de las frecuencias restantes.

Las datos obtenidos en cuanto a la aceptación de la CVA implementada con objetivos definidos (acercamiento intercultural y fortalecimiento de la lengua extranjera) fueron determinantes para asumir que los estudiantes se motivan cuando son parte de una comunidad, ante ello se puede establecer diferentes temáticas para implementar una 
CVA partiendo de los intereses y necesidades de los estudiantes y su finalidad es el aprendizaje mancomunado de sus miembros mediante la colaborción y la cooperación, esto se pudiera esbozar en una implementacion permanente no sólo de manera transversal sino que sea un estilo de aprendiaje desarrollando capacidades individuales y colectivas y que pueda alcanzar a toda la comunidad educativa de una institución.

\section{PROPUESTA}

Los resultados del proceso investigativo son decidores en cuanto a la incidencia positiva de una Comunidad Virtual de Aprendizaje y en base a dichos resultados la propuesta se direcciona a que las instituciones educativas puedan incorporar una CVA como proceso metodológico o como herramienta didáctica para propiciar aprendizajes en los estudiantes de manera participativa y con un sentido de pertenencia, todo eso conlleva a que se establezca pasos para implementar una CVA, tomando como referencia la manera en cómo se ejecutó y tomando los aspectos positivos para pulir el procedimiento a seguir siempre teniendo como horizonte el aprendizaje de los estudiantes que son parte de la comunidad y la cooperación entre los mismos a fin de sacar el mayor provecho de los beneficios de una CVA como parte del proceso educativo. 


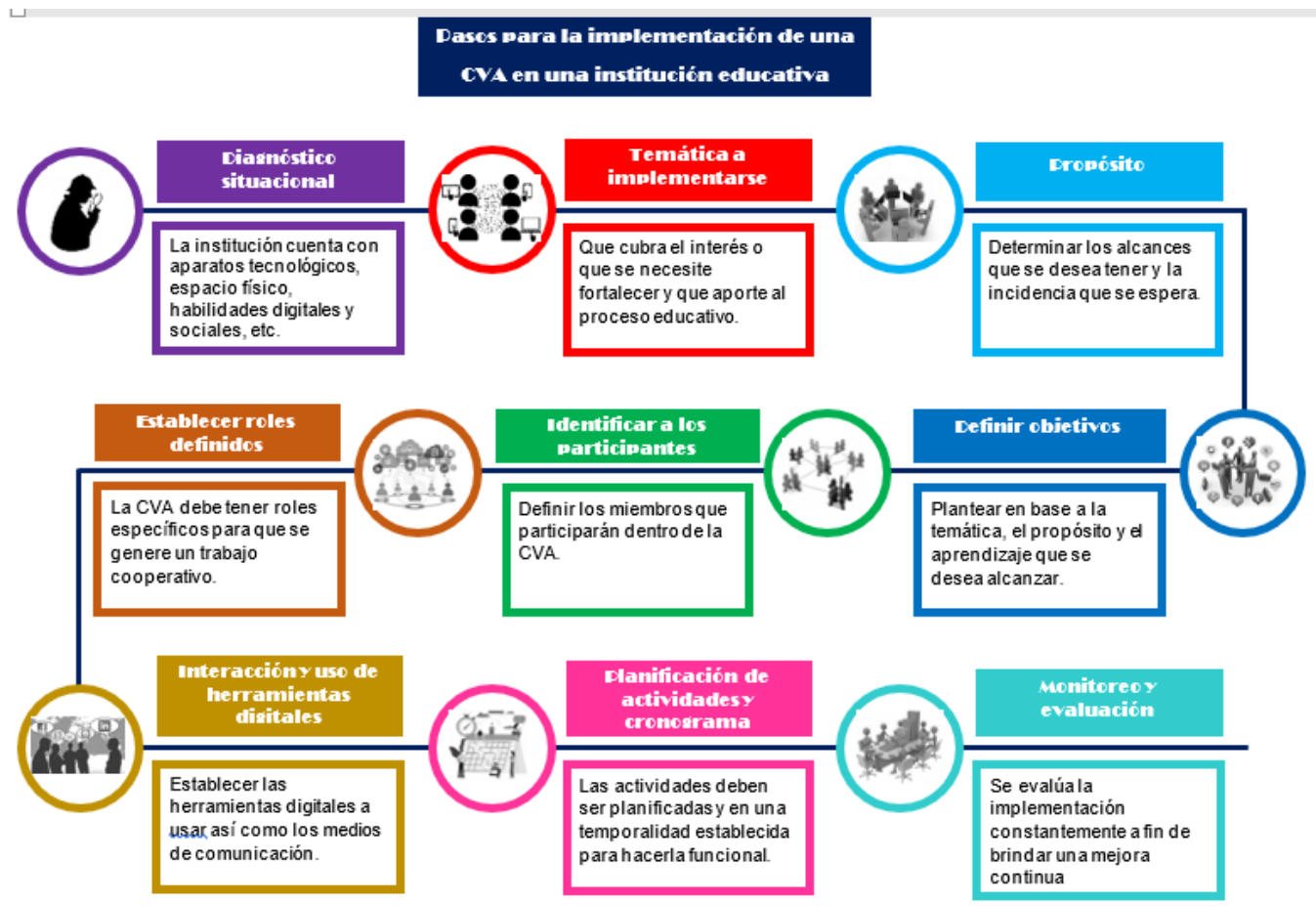

Figura 2. Propuesta de implementación de una CVA dentro de una institución educativa Elaboración: Los autores.

Ahondando los 9 pasos propuestos para la implementación y funcionalidad de una CVA se detalla en cada etapa lo siguiente:

Paso 1: Diagnóstico situacional, etapa en la que nos permite aclarar el panorama de la situación de la institución educativa, si cuenta con el equipamiento, el espacio físico, las habilidades tecnológicas y digitales, el empoderamiento de los participantes (tanto docentes como estudiantes), la colaboración de los actores educativos, el consentimiento de las autoridades, alianzas institucionales, entre otras. Situaciones que deben ser analizas para que se pueda implementar ya que no sólo basta la voluntad para ejecutarla 
sino también el acceso a los recursos y las posibilidades internas y externas de la institución educativa.

Paso 2: Temática a implementarse, se debe tener en cuenta los nudos críticos en el aprendizaje de los estudiantes y establecer la temática desde esa perspectiva, por ejemplo: se puede establecer una CVA de ciencias, de herramientas digitales, de matemática, etc. áreas en las que se necesite ser trabajadas desde otra perspectiva y que los estudiantes necesiten reforzar o afianzar, esto les motivará a participar y a estar inmersos en el aprendizaje de una manera activa.

Paso 3: Propósito, hace referencia al cómo se va a implementar una CVA, en esta etapa se debe detallar si se desea hacer entre instituciones de un mismo sector, sólo con estudiantes de un nivel, entre instituciones de diferente región o país, o simplemente con los estudiantes que deseen participar en la ejecución; en todos los casos puestos como ejemplo se debe motivar su participación constante desde principio a fin.

Paso 4: Definir objetivos, hace referencia al por qué y para qué se implementa una CVA, los objetivos deben estar inmersos al proceso educativo macro curricular, es decir, una CVA no actúa de manera aislada sino como parte del proceso de enseñanza-aprendizaje incluso pudiendo ser una estrategia de innovación dentro de las instituciones.

Paso 5: Identificar a los participantes, se debe definir a que grupo de estudiantes está dirigido la CVA, debido a que los temas y las actividades que se desarrollen deben estar acorde a su edad e intereses. Hace referencia a quiénes conformarán la CVA.

Paso 6: Establecer roles de actuación, una CVA se sirve del trabajo cooperativo, es por ello que se debe asignar roles de actuación, nombrar un coordinador general, una persona que se encargue de las herramientas digitales, otra para los procesos de interacción, otra para crear actividades; etc. Los roles estarán definidos dependiendo las necesidades de cada institución y la funcionalidad que se desea brindar. En este punto es importante manifestar que los docentes deben ser creadores de recursos y no sólo consumidores, ya que al crearlos estamos atendiendo a las necesidades e intereses de los participantes de la comunidad virtual, se debe evitar adaptar recursos duplicados de 
otras temáticas que, a veces, no se relacionan con el objetivo. La formación al profesorado debe ser permanente y los docentes deben estar en continua autoeducación sobre el tema.

Paso 7: Definir medios de interacción y herramientas digitales a emplear, esta etapa se debe acordar las herramientas digitales a usar y que sean las adecuadas para que posibiliten la interacción, se debe tener en cuenta que dichas herramientas deben ser de fácil acceso y familiarizadas por los miembros de la comunidad, paulatinamente se puede ir incorporando nuevas herramientas conforme se vaya adquiriendo las habilidades digitales de los participantes, con ello variamos los recursos y posibilitamos la renovación de los mismos para evitar la rutina o el uso excesivo de un mismo recurso. Parte de la innovación es ser generadores de recursos.

Paso 8: Planificación de actividades y cronograma a ejecutarse, en esta etapa involucra nuevamente el trabajo cooperativo ya que el grupo de docentes inmersos y con los roles establecidos deben planificar las actividades, una CVA no debe ser improvisada, sino debe cimentarse en una planificación que regule los conocimientos a impartirse y el accionar de los participantes, la planificación guiará el proceso educativo y los recursos a emplearse dentro de un tiempo definido. El cronograma debe ser establecido para que se determine una temporalidad y se cumpla los objetivos dentro de un plazo establecido, pero pudiendo ser flexible. Hacer referencia al cuándo y cómo se va a ejecutar la CVA.

Paso 9: Monitoreo y evaluación, la CVA debe estar en constante monitoreo, desde su inicio hasta un período después de concluir, esto con el fin de dar correctivos si fuese necesario y potencializar sus fortalezas. La evaluación se enfoca a la implementación y ejecución de la CVA, la evaluación permite brindar una mejora continua, es decir, no debe determinar si la CVA fue buena o mala, sino que se debe tomar los puntos débiles y mejorarlos a fin de que su implementación sea significativa. Una evaluación final de una CVA permite analizar si los pasos anteriores fueron ejecutados y si se cumplió con el propósito y los objetivos, esto permite estar innovando futuras CVA que puedan ser implementadas. 


\section{CONCLUSIONES}

La virtualidad aplicada a la educación ha trascendido en cuanto a las nuevas formas metodológicas que se puede incorporar, lo que implica cambios que la comunidad educativa debe asumir, en este punto, se mantienen aún una brecha digital en donde se espera que todos asuman esta responsabilidad pero sin brindar los medios para hacerlo, por ello se sigue teniendo una resistencia al cambio. La capacitación de los docentes debe ser constante, lo que dificulta es que maestros con años de experiencia conservan su práctica de enseñanza siendo herméticos a la hora de incorporar herramientas digitales, generando un desequilibrio educacional entre una clase con un docente que se sirve de los beneficios de las herramientas digitales y una clase con un docente con prácticas conservadoras de dictado y copiado y recursos de antaño. El compromiso de asumir la responsabilidad digital esta en cada uno, desde las acciones gubernamentales y el empoderamiento indivudual por asumir el reto digital.

En cuanto a la metodología, el hecho de que el docente esté relacionado directamente con el proceso investigativo y sea parte del mismo fuen determinante porque se pudo evienciar en cada etapa los avances y aceptación de una CVA implementada en la escuela Gabriela Mistral. Los padres de familia estuvieron enterados de la implementación, por ende, el apoyo recibido fue innegable a la hora de recolectar los datos debido a la colaboración brindada lo que permitió validar los datos sin mayor problema, paso importante para obtener datos reales y paramétricos que nos permitieron analizar la implementación y obtener resultados concretos y precisos. Por ello, socializar oportunamente la implementación de una CVA a la comunidad educativa es tan importante como la implementación misma porque todos los actores deben estár inmersos en las prácticas innovadoras.

Los resultados obtenidos fueron concluyentes para concebir que el trabajo cooperativo interacciona efectivamente con una CVA, dicho de otro modo, una CVA se nutre de la cooperación y el trabajo cooperativo posibilita una buena implementación de una CVA, pero se afianza con el sentido de pertenecia de sus miembros y su interacción. Los 
estudiantes que participaron respondieron de manera efectiva y dinámica siendo estimulante para que se generen aprendizajes, el hecho de aportar con su material para que los otros puedan verlo y aprender de él motivo la interacción. Explorar más allá de sus posibilidades con ayuda de la virtualidad ayudó a fomentar un aprendizaje vivencial, ya que pudieron conocer otras realidades y otras culturas para promover el respeto por la diversidad. Sin duda, el uso de las herramientas digitales y los recursos innovadores posibilitó el aprendizaje significativo, el sólo hecho aprender a usar los recursos disponibles en la CVA fue importante ya que fomentaron la autoeducación y la curiosidad. Otra situación que debe resaltarse es que los docentes pueden ser creadores de sus propios recursos, asi responden de manera directa con las necesidades y gustos de los estudiantes; no se ve mal que un docente consuma los recursos que las plataformas le brindan, pero es mejor ser generdores de productos porque contextualizan la realidad de su grupo de clase y eso es parte de innovar las prácticas educativas.

Dentro de las instituciones educativas es útil y necesario que se implemente una CVA encaminada a cubrir necesidades o problemáticas, es decir, su implementación coadyuvará a que dichas necesidades sean abordadas de manera significativa, lo que motivará que los participantes se integren por amplia gama de posibilidades que se puede ofrecer encaminada a cubrir sus intereses, una comunidad en donde se puede aprender interactuando y sin las presiones que la escuela formal esta acostumbrada a emplear, así se desarrolla habilidades sociales y digitales que servirán para afrontar su vida diaria y estar acorde a lo que la sociedad digital espera. Por ello se propone pasos para implementar una CVA, en cada etapa se debe considerar los nudos críticos en el aprendizaje de los estudiantes para que su ejecución tenga por objeto fortalecer las debilidades y potenciar las habilidades.

\section{FINANCIAMIENTO}

No monetario. 


\section{AGRADECIMIENTO}

A la Jefatura de Posgrados de la Universidad Católica de Cuenca por permitir el desarrollo y fomento de la investigación.

\section{REFERENCIAS CONSULTADAS}

Cabero-Almenara, J. (2006). Comunidades virtuales para el aprendizaje. Su utilización en la enseñanza [Virtual communities for learning. Your use in the teaching]. Edutec. Revista Electrónica De Tecnología Educativa, (20), a053. https://doi.org/10.21556/edutec.2006.20.510

Camacho, A. (2018). El docente como agente implicado en crear Comunidades Virtuales de Aprendizaje [The teacher as an agent involved in creating Virtual Learning Communities]. Revista En Ciencias Sociales y Humanidades Apoyadas Por Tecnologías, 7(14), 56-67.

Carlén, U. (2002). Typology of online learning communities [ Tipología de comunidades de aprendizaje en línea]. First International Conference on NetLearning 2002. https://n9.cl/qe77n

Carpy, C. (2012). Miradas históricas de la educación y la pedagogía [Historical views of education and pedagogy]. (1st ed.). Ediciones Díaz de Santos. https://n9.cl/6ea0x

Cueva-Gaibor, D. (2020). La tecnología educativa en tiempos de crisis [Educational technology in times of crisis]. Conrado, 16(74), 341-348.

Duarte-Duarte, J., \& Jurado, J. C. (2008). Los procesos pedagógicos y su relación con la convivencia escolar [Pedagogical processes and their relationship with school. Revista Colombiana de Educación,

(55). https://doi.org/10.17227/01203916.7571

Escorcia, R., \& Gutiérrez, A. (2009). La cooperación en educación: una visión organizativa de la escuela [Cooperation in Education: An Organizational Vision of the School]. Educación y Educadores, 12, 121-133. https://n9.cl/yl41s

Gairín, J. (2006). Las comunidades virtuales de aprendizaje [Virtual learning communities]. Educar, 37, 41-64. 
Johnson, D. W., \& Johnson, R. T. (2014). Cooperative Learning in 21st Century. [Aprendizaje cooperativo en el siglo XXI]. Anales de Psicología/Annals of Psychology, 30(3), 841-851. https://doi.org/10.6018/analesps.30.3.201241

Lamí-Rodríguez-del-Rey, L, Rodríguez-del-Rey-Rodríguez, M, \& Pérez-Fleites, M. (2016). Las comunidades virtuales de aprendizaje: sus orígenes [virtual learning communities: their origins]. Revista Universidad y Sociedad, 8(4), 93-101.

Locke, J. (2000). Conversation and community: Chat in a virtual world. Lynn Cherny. Stanford, CA: CSLI Publications, 1999. Pp. 369. Applied Psycholinguistics, 21(1), 152-155. doi:10.1017/S0142716400221073

MinEduc. (2012). Tecnologías de la Información y Comunicación aplicadas a la educación [Information and Communication Technologies applied to education]. https://n9.cl/ni9y9

Morales, C. (2016). Comunidades Virtuales de Aprendizaje en la escuela: Análisis de las interacciones sociales para el desarrollo de colectivos inteligentes [Virtual Learning Communities at school: Analysis of social interactions for the development of intelligent groups]. https://n9.cl/3/zxs

Moreira-Segura, C., \& Delgadillo-Espinoza, B. (2015). La virtualidad en los procesos educativos: reflexiones teóricas sobre su implementación [Virtuality in educational processes: theoretical reflections on its implementation]. Revista Tecnología En Marcha, 28(1), pág. 121-129. https://doi.org/10.18845/tm.v28i1.2196

Moriña, A. (2011). Aprendizaje cooperativo para una educación inclusiva: desarrollo del programa pac en un aula de educación primaria [Cooperative learning for inclusive education: developing the pac program in a primary education classroom]. Estudios Sobre Educacion, 21, 199-216. https://hdl.handle.net/10171/22625

Prensky, P. M. (2010). Nativos e Inmigrantes Digitales [Digital Natives and Immigrants]. https://n9.cl/qcs78

Sangrá, A. (2001). Enseñar y aprender en la virtualidad [Teach and learn in virtuality]. Educar, 28, 117-131.

Soriano, A. (2015). Educar para la virtualidad y la virtualidad para educar [Educate for virtuality and virtuality to educate]. Diálogos, 13, 19-31. https://doi.org/10.5377/dialogos.v0i13.2197 
Revista Arbitrada Interdisciplinaria KOINONIA

Año VI. Vol VI. N³. Edición Especial: Educación II. 2021

Hecho el depósito de Ley: FA2016000010 ISSN: 2542-3088

FUNDACIÓN KOINONIA (F.K). Santa Ana de Coro. Venezuela.

María Verónica Peralta-Ortiz; Darwin Gabriel García-Herrera; Sandra Elizabeth Mena-Clerque

Torres, L., Prieto, E., \& López, L. (2012). Entornos virtuales de enseñanza-aprendizaje. Evaluación del uso de las herramientas virtuales en el máster de educación para el desarroll [Virtual environments for teaching-learning. Evaluation of the use of virtual tools in the master of education for su. Revista Electrónica de Tecnología Educativa, 39, 1-18. https://doi.org/https://doi.org/10.21556/edutec.2012.39.376

C2021 por los autores. Este artículo es de acceso abierto y distribuido según los términos y condiciones de la licencia Creative Commons Atribución-NoComercial-Compartirlgual 4.0 Internacional (CC BY-NC-SA 4.0)

(https://creativecommons.org/licenses/by-nc-sa/4.0/). 